\title{
Análisis Del Efecto De La Adición De Fibra Cítrica Del Bagazo De La Naranja En Las Propiedades Nutrimentales Y Sensoriales De Un Embutido Y Determinación De La Calidad Microbiológica
}

\author{
Temani Durán-Mendoza, \\ División Académica Multidisciplinaria de los Ríos. \\ Universidad Juárez Autónoma de Tabasco. Tabasco, México. \\ Manuel González-Pérez, \\ Universidad Popular Autónoma del Estado de Puebla (UPAEP). \\ Posgrado en Ciencias de la Ingeniería Biomédica. \\ Sistema Nacional de Investigadores Nivel 1. \\ María Concepción de la Cruz-Leyva, \\ José Ulises González-de la Cruz, \\ Carlos Alberto Cuenca-Soria, \\ Juan Guzmán-Ceferino, \\ División Académica Multidisciplinaria de los Ríos. \\ Universidad Juárez Autónoma de Tabasco. Tabasco, México. \\ Jesús Ofelia Angulo-Guerrero, \\ Instituto Tecnológico de Veracruz (ITV). \\ Sistema Nacional de Investigadores Nivel 2. \\ Laboratorio de Evaluación Sensorial. Unidad de Investigación y \\ Desarrollo en Alimentos Veracruz, México. \\ Carolina del Carmen Pérez-Sánchez, \\ División Académica Multidisciplinaria de los Ríos. \\ Universidad Juárez Autónoma de Tabasco. Tabasco, México.
}

Doi: 10.19044/esj.2018.v14n18p14 URL:http://dx.doi.org/10.19044/esj.2018.v14n18p14

\begin{abstract}
The orange bagasse (Citrus sinensis) is a residue with a significant content of citrus fiber (FC); it is used as a functional ingredient in the development of new products. We consider it essential to know the effect of the nutritional and sensorial properties of FC. The objective of this research was to evaluate the effect of powdered the orange bagasse on the nutritional and sensorial properties of pork and beef sausage. The sausage was prepared by mixing the proportions of minced pork and beef with the corresponding
\end{abstract}


FC, adding spices, orange juice and a solution of natural dye Bixa Orellana, after 24 hours of maturation was stuffed into cellulose casings. A randomized design with three repetitions was used. The treatments were formulations with 0, 5, 10 and $15 \%$ of FC respectively, named treatments E0, E5, E10 and E15. The chemical variables evaluated were: moisture content, ash, fat, protein (analyzed by the AOAC method, 1990), and total dietary fiber (by the methodology described by Asp et al., 1983 and Prosky et al, 1988); the microbiological variables: aerobic mesophiles, total and fecal coliforms, Salmonella and Staphylococcus aureus, detected and quantified with the methods of the corresponding Official Mexican Standards. The sensory variable was the level of liking which was evaluated in 128 consumers, and a commercial sausage called "EC" was used. As a comparison product. In E5, E10 and E15 the fat levels decreased ( $<<0.05)$. The ash increased as a function of the addition of FC. Regarding protein, no differences were observed between E0 and E5. In the treatments, E10 and E 15 the protein decreased significantly. Total dietary fiber at E5, E10, and E15 ranged from 4.38 to $10.50 \%$. The consumer test favored E0, E5, and EC. We conclude that the orange bagasse exerts a positive effect by decreasing fat and increasing total dietary fiber. Aleson-Carbonell et al. (2003) report similar behavior. It is concluded that it is feasible to add FC no higher than 5\% as an alternative to enrich sausages.

Keywords: Citrus fiber, Bagasse of the orange, nutritional and sensorial properties, sausage, microbiological quality

\section{Resumen}

El bagazo de naranja (Citrus sinensis) es un residuo con un contenido importante de fibra cítrica (FC); ésta es utilizada como un ingrediente funcional en el desarrollo de nuevos productos. Nosotros consideramos importante conocer el efecto de las propiedades nutritivas y sensoriales de la FC. El objetivo de esta investigación fue evaluar el efecto del bagazo de naranja pulverizado en las propiedades nutritivas y sensoriales de un embutido de carne de cerdo y res. El embutido se elaboró mezclando las proporciones de carne picada de cerdo y res con la FC correspondiente, añadiendo especias, jugo de naranja y una solución de colorante natural de Bixa Orellana, después de 24 horas de maduración se embutió en tripas de celulosa. Se empleó un diseño aleatorio con tres repeticiones. Los tratamientos fueron formulaciones con $0,5,10$ y $15 \%$ de FC respectivamente denominados tratamientos E0, E5, E10 y E15. Las variables químicas evaluadas fueron: el contenido de humedad, ceniza, grasa, proteína (analizadas mediante los método del AOAC, 1990) y fibra dietética total (mediante la metodología descrita por Asp et al., 1983 y Prosky et al, 1988); las variables microbiológicas: mesófilos aerobios, 
coliformes totales y fecales, Salmonella y Staphylococcus aureus, detectadas y cuantificadas con los métodos de las Normas Oficiales Mexicanas correspondiente. La variable sensorial fue el nivel de agrado la cual se evaluó en 128 consumidores y se utilizó un embutido comercial denominado EC, como producto de comparación. En E5, E10 y E15 los niveles de grasa disminuyeron $(\mathrm{p}<0,05)$. La ceniza aumentó en función de la adición de la fibra. En cuanto a proteína, no se observó diferencias entre E0 y E5. En los tratamientos E10 y E15 disminuyó la proteína significativamente. La fibra dietética total en E5, E10 y E15 fluctuó de 4,38 a 10,50 \%. La prueba de consumidores favoreció a E0, E5 y EC. Nosotros concluimos que la FC ejerce un efecto positivo al disminuir la grasa y aumentar la fibra dietética total. Aleson-Carbonell et al. (2003) reportan un comportamiento similar. Se concluye que es factible la adición de la fibra cítrica no mayor a $5 \%$ como alternativa para enriquecer embutidos.

Palabras claves: Fibra cítrica, Bagazo de la naranja, propiedades nutrimentales y sensoriales, embutido, calidad microbiológica

\section{Introducción}

Trejo-Marquez et al. (2017); Martínez et al. (2008) utilizaron los desechos de frutas, hortalizas y otros vegetales como fuentes alternas de fibra dietética; ésta ha sido muy estudiada ya que ofrece diversos beneficios a la salud como prevenir la diabetes, cardiopatía o neoplasias del estómago, aligeramiento del tránsito intestinal, eliminación de grasas, entre otras. Además, al presentar propiedades funcionales como: la capacidad de hinchamiento, la capacidad de retención de agua y la capacidad de retención de aceite, puede ser explotadas para el desarrollo de nuevos productos alimenticios, brindándole nuevas y mejores características al producto final.

Por otro lado, Fernández-López y et al. (2003) utilizaron fibra de cítricos en productos cárnicos, donde la presencia de fibra dietética y antioxidantes de subproductos de frutas cítricas permitieron la producción de un alimento más sano, que supuestamente ayudara a superar el déficit de la fibra en la dieta humana y a prevenir el desarrollo de enfermedades asociadas a la pobre ingesta de fibra. Además, Martínez et al. (2003) realizó a través de pruebas descriptivas la caracterización sensorial de fibras de naranja (Citrus sinensis), mandarina (Citrus reticulata), limón (Citrus limon), piña (Ananas comosus), maracuyá (Passiflora edulis) y mango (Mangifera indica), con el fin de elaborar el perfil de sabor y el perfil de textura para proceder a hacer las formulaciones de productos que satisfagan a los consumidores.

Algunos investigadores utilizaron un panel no entrenado de 40 personas para determinar el efecto de la incorporación de la harina de cáscara 
de naranja, el cual mostró que no hubo diferencias significativas entre la muestra control y la elaborada con harina de cáscara de naranja.

El objetivo de esta investigación fue evaluar el efecto de la adición de fibra cítrica del bagazo de la naranja en las propiedades nutrimentales y sensoriales de un embutido y determinar la calidad microbiológico del producto cárnico.

\section{Materiales y métodos}

\section{Obtención de la fibra procedente del bagazo de naranja.}

Las naranjas (Citrus sinensis, procedente del municipio de Martínez de la Torre, Veracruz, México) se lavaron con abundante agua y detergente. Se les eliminó la cáscara con una peladora mecánica de naranjas (mca. Gimbel Mexicana S.A de C.V). Se extrajo el jugo con un extractor Citromatic (mca. Braun) y se eliminaron manualmente las semillas. El bagazo se cortó en triángulos de 2 x $3 \mathrm{~cm}$ y se pesó. Para eliminar la limonina (sustancia responsable del amargor) se sometió dos veces a un tratamiento térmico a 95 ${ }^{\circ} \mathrm{C}$ durante 30 minutos en una solución con $\mathrm{NaCl}$ al 15\%. Al término de cada tratamiento, el bagazo se lavó cinco veces con abundante agua fría para retirar el exceso de $\mathrm{NaCl}$ y se filtró. El producto obtenido se secó a $60{ }^{\circ} \mathrm{C}$ durante 6 h en un secador de charola (mca. Apex. Tipo SSE 17M, Serie A3985414.) Se trituró en un molino de corte (mca. Glen Creston, Serie 011158) con un tamiz No. $0.25 \mathrm{~mm}$, para obtener la fibra pulverizada. Finalmente se almacenó a $20{ }^{\circ} \mathrm{C}$ hasta su utilización.

\section{Elaboración del embutido cárnico (longaniza) adicionado con fibra.}

Se prepararon tres formulaciones del embutido con fibra dietética al $5 \%, 10 \%$ y $15 \%$, y una formulación testigo con $0 \%$ de fibra, denominadas como E5, E10, E15 y E0, respectivamente. La proporción de la carne de cerdo, carne de res, grasa mixta y fibra dietética se muestra en la tabla 1.

El embutido se elaboró de la siguiente manera: se mezcló la carne molida de res y cerdo con la grasa mixta y la fibra de naranja. Se agregó una solución de colorante natural procedente de Bixa Orellana (achiote) (en 300 $\mathrm{mL}$ de jugo de naranja agria se diluyó $4 \mathrm{~g}$ de achiote por cada $1000 \mathrm{~g}$ de producto) y se incorporaron las especias en polvo. Se homogenizó manualmente la mezcla durante 5 minutos y se dejó reposar durante 24 horas a $8 \pm 3{ }^{\circ} \mathrm{C}$. Posteriormente la mezcla de carne madurada se embutió en tripas de celulosa (calibre $2 \mathrm{~cm}$ de diámetro). Finalmente, el producto se almacenó en a $8 \pm 3{ }^{\circ} \mathrm{C}$ hasta su utilización. 
Tabla 1. Formulaciones experimentales de los embutidos.

\begin{tabular}{lcccc}
\hline \hline \multirow{2}{*}{ INGREDIENTES } & \multicolumn{4}{c}{ EMBUTIDOS } \\
\cline { 2 - 5 } & E0 & E5 & E10 & E15 \\
\hline Carne de cerdo & $60 \%$ & $55 \%$ & $50 \%$ & $45 \%$ \\
Carne de res & $20 \%$ & $20 \%$ & $20 \%$ & $20 \%$ \\
Grasa mixta & $20 \%$ & $20 \%$ & $20 \%$ & $20 \%$ \\
Fibra de naranja & $0 \%$ & $5 \%$ & $10 \%$ & $15 \%$ \\
\hline \hline
\end{tabular}

\section{Análisis bromatológicos}

Los métodos empleados para los análisis bromatológicos se indican en la tabla 2, estos se efectuaron por triplicado en cada formulación.

Tabla 2. Métodos de análisis químico.

\begin{tabular}{ll}
\hline \hline \multicolumn{1}{c}{ ANÁLISIS } & \multicolumn{1}{c}{ MÉTODO DE DIAGNÓSTICO } \\
\hline Humedad. & AOAC (1990) No. 950.46B \\
Cenizas. & AOAC (1990) No. 942.05 \\
Extracto etéreo. & AOAC (1990) No. 960.39 y 920.39C \\
Proteína. & AOAC (1990) No.981.10 \\
Fibra dietética total, FDS, FDI. & Asp et al., (1983) y Prosky et al, (1988) \\
\hline \hline
\end{tabular}

Para determinar la fibra dietética total, la fibra dietética soluble e insoluble (FDT, FDS y FDI), el método enzimático-gravimétrico empleado recomienda que para la hidrólisis del almidón se utilice $\alpha$-amilasa y amiloglucosidasa. Esta no se realizó, debido a que este tipo de producto cárnico no tiene como ingrediente almidón. Sin embargo, se examinó en los tratamientos la posible contribución de almidón por parte del bagazo de naranja. Para esto, se realizó una prueba de almidón cualitativa por el método 925.38 descrito por el AOAC (1990). Al comprobar que el bagazo de naranja y los productos cárnicos no contenían almidón se procedió a hacer la determinación sin las enzimas amilolíticas. La hidrólisis proteolítica se efectuó con la enzima proteasa de (Bacillus licheniformis) (mca. Sigma-Aldrich P3910).

\section{Análisis microbiológicos.}

Los métodos empleados para detectar y cuantificar la presencia de los microorganismos se muestran en la tabla 3.

Tabla 3. Métodos de análisis microbiológico.

\begin{tabular}{lc}
\hline \multicolumn{1}{c}{ ANÁLISIS } & MÉTODO DE DIAGNÓSTICO \\
\hline Manejo y preparación de las muestras. & NOM-110-SSA1-1994 \\
Determinación de mesofílicos aerobios. & NOM-092-SSA1-1994 \\
Determinación de coliformes totales y fecales. & NOM-112-SSA1-1994 \\
Determinación de Salmonella. & NOM-114-SSA1-1994 \\
Determinación de Staphylococcus aureus. & NOM-115-SSA1-1994 \\
\hline \hline
\end{tabular}




\section{Prueba sensorial de consumidores (prueba hedónica)}

Para conocer el nivel de agrado de los productos formulados se efectuó una prueba de tipo afectiva: la prueba hedónica, la cual se aplicó con el procedimiento Rank-Rating descrito por O’Mahony (1999). Se solicitó la opinión individualizada de un total de 128 posibles consumidores, los cuales fueron elegidos al azar en un establecimiento comercial. Los cuatro embutidos experimentales (E0, E5, E10 y E15) fueron comparados contra uno comercial (EC). Las muestras fueron preparadas de la siguiente manera: se eliminó la tripa de celulosa de los embutidos y se sometió a cocción (en un recipiente de teflón antiadherente) durante 10 minutos, se envolvió $10 \mathrm{~g}$ de muestra en papel aluminio $(10 \times 10 \mathrm{~cm})$. Las muestras ya cocidas fueron transportadas al centro comercial y de nuevo se calentaban al vapor durante 5 minutos a $90{ }^{\circ} \mathrm{C}$ en una freidora eléctrica (mca. Taurus Mexicana Modelo FC-1B). La temperatura de consumo fue de $45 \pm 2{ }^{\circ} \mathrm{C}$. Entre cada muestra el juez eliminaba el sabor residual de producto anterior con totopos de maíz y agua.

\section{Análisis estadísticos}

Los resultados de los análisis proximales se examinaron mediante el ANOVA y una prueba de Tukey-Kramer HSD $(P<0.05)$. Los resultados de los análisis microbiológicos fueron interpretados en base a las especificaciones sanitarias por la NOM-213-SSA1-2002 y la NOM-034SSA1-1993. Los resultados de la prueba de consumidores (hedónica) fueron examinados a través del ANOVA y una prueba de Fisher LSD $(P<0.05)$, y se utilizó la moda como medida de tendencia central. Los análisis estadísticos se efectuaron con el software JMP 6.0 (del SAS).

\section{Resultados}

\section{Análisis bromatológicos.}

Los resultados de la composición bromatológica de los embutidos se presentan en la tabla 4.

Tabla 4. Composición bromatológica de los embutidos elaborados.

\begin{tabular}{lcllr}
\hline \hline \multicolumn{2}{c}{ E0 } & E5 & \multicolumn{1}{c}{ E10 } & \multicolumn{1}{c}{ E15 } \\
\hline HUMEDAD (\%) & $59.547 \pm 0.262^{\mathrm{a}}$ & $60.420 \pm 0.274^{\mathrm{b}}$ & $59.172 \pm 0.149^{\mathrm{a}}$ & $55.647 \pm 0.097^{\mathrm{c}}$ \\
CENIZAS (\%) & $2.712 \pm 0.083^{\mathrm{a}}$ & $2.982 \pm 0.098^{\mathrm{b}}$ & $3.418 \pm 0.093^{\mathrm{c}}$ & $3.747 \pm 0.075^{\mathrm{d}}$ \\
LÍPIDOS (\%) & $18.876 \pm 0.132^{\mathrm{a}}$ & $16.998 \pm 0.259^{\mathrm{bc}}$ & $16.486 \pm 0.160^{\mathrm{c}}$ & $17.068 \pm 0.255^{\mathrm{b}}$ \\
PROTEINA (\%) & $14.569 \pm 0.357^{\mathrm{a}}$ & $13.540 \pm 0.604^{\mathrm{ab}}$ & $12.272 \pm 0.185^{\mathrm{bc}}$ & $11.040 \pm 0.808^{\mathrm{c}}$ \\
FDT (\%) & $0.596 \pm 0.005^{\mathrm{a}}$ & $4.381 \pm 0.291^{\mathrm{b}}$ & $7.967 \pm 0.034^{\mathrm{c}}$ & $10.498 \pm 0.300^{\mathrm{d}}$ \\
FDI (\%) & $0.078 \pm 0.006^{\mathrm{a}}$ & $2.560 \pm 0.388^{\mathrm{b}}$ & $4.823 \pm 0.197^{\mathrm{c}}$ & $6.240 \pm 0.029^{\mathrm{d}}$ \\
FDS (\%) & $0.518 \pm 0.009^{\mathrm{a}}$ & $1.821 \pm 0.224^{\mathrm{b}}$ & $3.144 \pm 0.167^{\mathrm{c}}$ & $4.258 \pm 0.329^{\mathrm{d}}$ \\
\hline \hline
\end{tabular}

Los valores presentados son los promedios \pm desviaciones estándar, (n $=3$ ). 
Letras diferentes en una misma fila indican diferencias estadísticamente significativas de acuerdo con la prueba de Tukey $(P<0.05)$.

El pulverizado de fibra obtenido del bagazo de naranja tuvo una concentración de FDI de $41.75 \% \pm 0.95$ y de FDS de $26.50 \% \pm 1.0$.

\section{Análisis microbiológicos}

Los resultados de los análisis microbiológicos se muestran en la tabla 5. Tabla 5. Resultados de los análisis microbiológicos.

\begin{tabular}{lccc}
\hline \hline MICROORGANISMO & E0 & E5 & E10 \\
\hline Mesofílicos (UFC/g) & $1.0 \times 10^{-5}$ & $3.0 \times 10^{-5}$ & $2.5 \times 10^{-5}$ \\
C. Totales (NMP/g) & 34 & 23 & 43 \\
C. Fecales (NMP/g) & 2 & 3 & 15 \\
Salmonella en 25g de muestra & Negativo & Negativo & Negativo \\
S. aureus en 25g de muestra & Negativo & Negativo & Negativo \\
\hline \hline
\end{tabular}

\section{Discusión}

\section{Análisis bromatológicos}

El resultado de FDI en el bagazo de la naranja concuerda con las concentraciones obtenidas por Chau y Haung (2003) y Rincón et al. (2005) quienes reportan una concentración de $47.6 \% \pm 10.8$ y 49.78\%, respectivamente. En cuanto a la FDS estos autores reportan una menor concentración en comparación con la determinada en esta investigación, esto puede deberse a los diferentes procesos de preparación del pulverizado de fibra, ya que Larraurri et al. (1997) reporta un contenido de 21-22\% de FDS en la cáscara naranja.

La humedad de los embutidos adicionados con fibra dietética fue mayor al del testigo, esto puede deberse a que la fibra insoluble del bagazo de naranja tiene una alta capacidad de absorción de agua, Chau y Haung (2003) demostraron que ésta es de 15.5 - $16.7 \mathrm{~mL} / \mathrm{g}$, esta característica es lo que posiblemente favorece este incremento de humedad en los embutidos E5, E10 y E15. Comportamientos semejantes fueron reportados por Aleson-Carbonell et al. (2003) quienes adicionaron fibra del bagazo del limón en salchichas, el contenido de humedad de los productos con fibra fue mayor con respecto al producto sin fibra.

Se registró una disminución significativa de lípidos en los embutidos E5, E10 y E15 con respecto a E0. Estos resultados confirman que la adición de fibra tiene un efecto positivo al disminuir significativamente los niveles de grasa en este tipo de productos altamente calóricos. Aleson-Carbonell et al. (2003) reportan un comportamiento similar.

En cuanto al contenido de proteína no existe diferencias $(P<0.05)$ entre E5 y E0, pero sí se observa diferencias entre E10 y E15 con respecto a E0; la concentración de proteína en E10 y E15 (12.27\% y 11.04\%) está por 
debajo del contenido normal, lo cual lo indica que la adición de un 15\% y $10 \%$ del pulverizado de fibra del bagazo no es recomendada en estos productos, pues de acuerdo con las tablas de alimentos el contenido de proteína en las longanizas debe ser de 16.6\% - 13.4\% (FAO/LATINFOODS, 2002). Otra razón por la que no se recomienda elaborar el embutido E15 es que durante el proceso de elaboración se presentó dificultad para homogenizar adecuadamente la mezcla de ingredientes y durante el proceso de embutido se mantuvo muy rígida, lo que provocaba que la tripa se reventara.

El contenido de FDT presente en E5, E10 y E15, fue de 4.38\%, 7.97\% y $10.50 \%$, respectivamente. Cabe notar que el embutido testigo (T0) contiene un $0.60 \%$ de FDT; esto posiblemente se debe a que durante el proceso de elaboración de las longanizas se utiliza jugo de naranja agria (Citrus aurantium var. amara L) para madurar el producto en un medio ácido, este ingrediente contiene fibra soluble, y es lo que aporta junto con las especias (en menor proporción) la fibra a E0.

La carga de bacterias mesofílicas aerobias en los embutidos están dentro del límite permisible por la NOM-034-SSA1-1993, la cual especifica un límite máximo de $5.0 \times 10^{-5} \mathrm{UFC} / \mathrm{g}$ de carne molida cruda. La presencia de coliformes en alimentos resulta de la exposición de estos al medio ambiente (tierra, aire, residuos en utensilios o equipos, insectos, etc.), y la tolerancia en alimentos está en función del tipo de producto. Por otro lado, en ningún tratamiento se detectó la presencia de Salmonella y de Staphylococcus aureus, por lo que se cumple la NOM-213-SSA1-2002, lo que indica que los productos elaborados no representarían un riesgo a la salud de los consumidores, en caso de que el producto no recibiera un tratamiento térmico adecuado.

\section{Prueba sensorial de consumidores (prueba hedónica)}

Los resultados de la prueba de consumidores se muestran en la tabla 6 , y figura 1. En EC el nivel de agrado según la moda fue de 8 y 6 en la escala hedónica, esto indica que la mayoría de los consumidores la califican como un producto que gusta mucho y gusta poco; E0 y E5 tuvieron una moda de 7, es decir que el producto elaborado gusta moderadamente; los embutidos con mayor adición de fibra (E0 y E15) gustaron poco, la moda fue de 6 (figura 1). De las tres formulaciones adicionadas con fibra, E5 fue el que tuvo un mayor agrado entre los consumidores, este fue calificado similar a los dos productos controles EC y E0 (tabla 6 y figura 1). Estos resultados sugieren la factibilidad de adicionar un 5\% (4.38\% de FDT) de pulverizado de fibra de naranja en los embutidos, esto debido a que no tiene efecto negativo en la palatabilidad del alimento.

Tabla 6. Comparación del nivel de agrado de los productos.

LONGANIZAS NIVEL DE AGRADO

Embutido comercial (EC) $6.2 \pm 2.2^{\mathrm{ab}}$ 
Embutido testigo (E0)

Embutido con fibra (E5)

Embutido con fibra (E10)

Embutido con fibra (E15)

$$
\begin{gathered}
6.4 \pm 1.6^{\mathrm{a}} \\
6.4 \pm 1.6^{\mathrm{a}} \\
5.8 \pm 1.8^{\mathrm{bc}} \\
5.5 \pm 1.7^{\mathrm{c}}
\end{gathered}
$$
$=128$ ).

Los valores presentados son los promedios \pm desviaciones estándar, (n

Letras diferentes en el sentido de las columnas indican diferencias estadísticamente significativas de acuerdo con la prueba de Fisher LSD $(P<0.05)$.

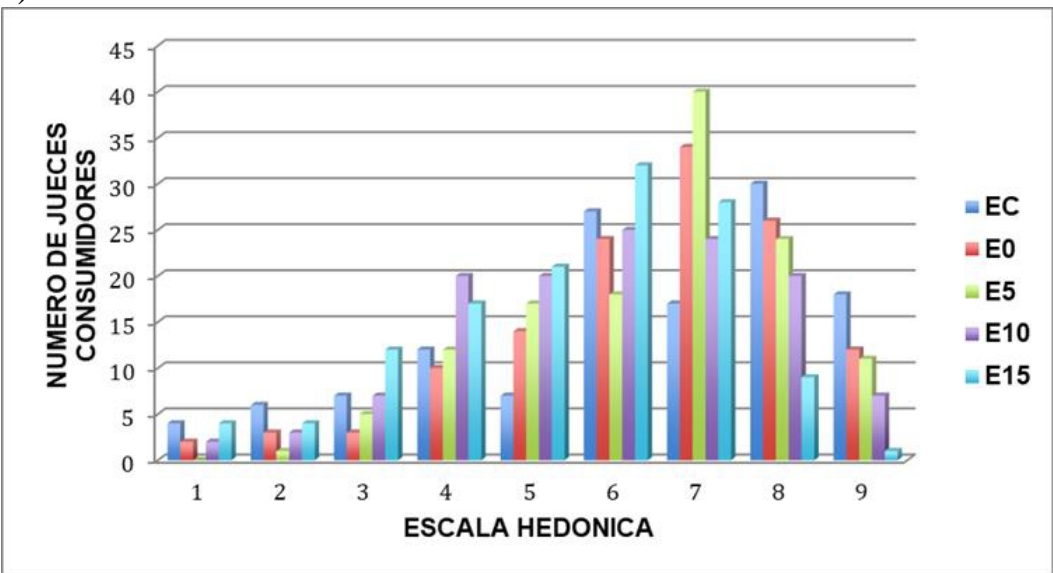

Figura 1. Gráfica de frecuencia de los resultados de la prueba hedónica

\section{Conclusion}

El bagazo de naranja pulverizado demostró ser una buena fuente de fibra dietética para el enriquecimiento de embutidos cárnicos cuando se incorpora en una concentración del 5\%; sin embargo, en concentraciones del $10 \%$ y $15 \%$ no es recomendable, ya que afecta significativamente sus propiedades químicas, microbiológicas y sensoriales. Estos resultados sugieren que el contenido de fibra de $4.38 \%$ no tiene un efecto negativo en la palatabilidad del alimento. Además, con la formulación E5 se contribuye con un $14.6 \%$ de fibra en la IDR.

\section{References:}

1. Pino, J. A., Hall, Y. C., Reynaldo, L. S., \& Enríquez, I. I. (2018). Caracterización de compuestos volátiles en néctares de acerola, guayaba y acerola-guayaba. Ciencia y Tecnología de Alimentos, 27(2).

2. Aleson-Carbonell L., Fernández-López J., Sayas-Barberá E., Sendra E., Pérez-Alvarez J.A. (2003). Utilization of Lemon Albedo in Drycured Sausages. J. Food Sci 68(5): 1826-1830. 
3. AOAC. (1990). Official methods of analysis of the association of official analytical chemists. 15 th edition. Arlington Virginia. USA. Pp. 70, 79, 922, 931 y 937.

4. Asp N.G., Johansson G.C., Hallmer H., Siljeström M. (1983). Rapid enzymatic assay of insoluble and soluble dietary fiber. J. Agric. Food Chem. 31(3):476-482.

5. Chau C.F and Huang Y.L. (2003). Comparison of the chemical composition and physicochemical properties of different fibers prepared from the peel of Citrus sinensis L.Cv, Liucheng. J. Agric. Food Chem 51(9) 2615-2618.

6. FAO/LATINFOODS. (2002). Tabla de Composición de Alimentos de América Latina. Longaniza, Código. F243 y F244. Disponible en la World Wide Web: http://www.rlc.fao.org/bases/alimento. Fecha de consulta 5/10/2007.

7. Fernandez-Lopez J., Fernandez-Gines J.M., Aleson-Carbonell L, Sendra E., Sayas-Barbera E, Perez-Alvarez Ja. (2003). Application of functional citrus by-products to meat products. Trends in food Science and Technology 15:176-185.

8. Larrauri A.J., Borroto B., Crespo R.A. (1997). Water recycling in processing orange peel to a high dietary fibre powder. International Journal of Food Science and Technology. 1997, 32, 73-76.

9. Martínez A, Olga L, Román M, María O, Gutiérrez E, Ester L, Medina M, Gilma B, Cadavid C, Margarita, \& Flórez A, Óscar A. (2008). Desarrollo y evaluación de un postre lácteo con fibra de naranja. Vitae, 15(2)

10. Martínez, O. L., Roman, M. O., Gutiérrez, E. L., Medina, G. B., \& Flórez, O. A. (2003). Caracterización sensorial de fibras de algunas frutas comunes en Colombia. Vitae, 10(2).

11. Normas Oficiales Mexicanas: NOM-034-SSA1-1993. Bienes y servicios. Productos de la carne. Carne molida y carne molida moldeada. Envasadas. Especificaciones sanitarias; NOM-092-SSA11994. Bienes y Servicios. Métodos para la cuenta de bacterias aerobias en placa; NOM-110-SSA1-1994. Bienes y Servicios. Preparación y dilución de muestras de alimentos para su análisis microbiológico; NOM-112-SSA1-1994. Bienes y Servicios. Determinación de bacterias coliformes. Técnicas del número más probable; NOM-114SSA1-1994. Bienes y Servicios. Método para la determinación de salmonella en alimentos; NOM-115-SSA1-1994. Bienes y Servicios. Método para la determinación de Staphylococcus aureus en alimentos; NOM-213-SSA1-2002. Productos y Servicios. Productos cárnicos procesados. Especificaciones sanitarias. Método de prueba. 
12. O’Mahony M. (1999). Food sensory science. Department of food science and technology. University of California, Davis, California. Pp.163F, 163G, 163H y 163I.

13. Prosky L., Asp N.G., Schweizer T, Devries J., Furda I. (1988). Dertermination of insoluble, soluble and total dietary fiber in foods product; interlaboratory study. J Assoc of Anal Chem. 71(5):10171023.

14. Rincón A.M., Vasquez A.M., Padilla C.F. (2005). Composición química y compuestos bioactivos de las harinas de cáscaras de naranja (Citrus sinensis), mandarina (Citrus reticulata) y toronja (Citrus paradisi) cultivadas en Venezuela. Archivos Latinoamericanos de Nutrición. 55(3):305-310.

15. Trejo Márquez, M. A., Lira-Vargas, A. A., \& Pascual-Bustamante, S. (2017). Fibra para el futuro: Propiedades y beneficios. OmniaScience Monographs. 